\title{
Research on Node Context Model for the Environmental Parameters of Standard Chicken Henhouse
}

\author{
Pu Haitao, Zhang Aiying, Song Enzhao, and Lin Jinjiao
}

\begin{abstract}
Aiming at the issue of time consuming excess on single node during some workflow activities for the environmental parameters of standard chicken henhouse, this paper builds a P2P network node context based model on task allocating, scheduling and solving. Focusing on the dynamic characteristic of P2P network environment, the model adopts node context to describe the workflow environment. The workflow management system can obtain the environmental dynamic changes through perceiving context information, and be used to manipulate task allocating and scheduling. Basing on the node context, this paper presents the node handling ability assessment index, with which the workflow management system can calculate out the price index and node task allocating index that equles to execution time and manipulate the task scheduling.
\end{abstract}

Index Terms-Context, standard chicken henhouse, node model.

\section{INTRODUCTION}

It is more and more care for the environmental parameters of standard chicken henhouse. In1982, Drury and Mitchell developed the environmental control system [1]-[3]. It is founded that task allocating and scheduling is a NP problem, so it is a feasible way to find optimal result in polynomial time by using heuristic algorithm [4]. But in P2P network [5], it is hard to estimate precisely the task handling time of every node in advance. So the global environment and static information based heuristic algorithm [6], [7] can hardly work on solving real time task allocation. Aiming at the issue of time consuming excess on single node during some workflow activies for the environmental parameters of standard chicken henhouse, this paper builds a P2P network node context based model on task allocating, scheduling and solving. Focusing on the dynamic characteristic of P2P network environment, the model adopts node context to describe the workflow

Manuscript received September 17, 2013; revised November 18, 2013 This work has been supported by the Shandong Science and Technology Development Project (2012GGX10122), the Shandong Province Higher Educational Science and Technology Program (J10LG16; J11LG21 and J13LN35), the Shandong Doctoral Foundation (BS2009DX039), Jinan Science and Technology Development Project (201202058) and Teaching Reform of the Key Project of Shandong Province (2012073).

$\mathrm{Pu}$ Haitao is with the Department of Electrical Engineering \&Information Technology, Shandong University of Science and Technology, Jinan, 250031, P.R.China (e-mail: pht@sdust.edu.cn).

Zhang Aiying is with Qingdao Agriculture University, Qingdao, 266109, P.R.China (e-mail: zhangay01@163.com).

Song Enzhao is with Beijing Jiaotong University, Beijing, 100044, P.R.China (e-mail: philipjinan@163.com).

Lin Jinjiao is with the School of Management Science and Engineering, Shandong University of Finance and Economics, Jinan, 250031, P.R.China (e-mail: linjj112@163.com). environment [8], [9]. The workflow management system can obtain the environmental dynamic changes through perceiving context information, and be used to manipulate task allocating and scheduling. Basing on the node context, this paper presents the node handling ability assessment index, with which the workflow management system can calculate out the price index and node task allocating index that equles to execution time and manipulate the task scheduling.

\section{CAN NODE CONTEXT}

CAN ( Content Addressing Network) is a typical center free structured P2P network, it has applications in a wide range of fields because of its suitable complexity of routing algorithm, simple structure, great maintenability and reliability.

Context includes a group of present applications relating states and variables, some of which can alter the system behaviors directly and the others can affect system behaviors by users through attracting the interes of users, in the computer system environment. P2P network node context is a set of device-dependent, software management integrated physical resource parameters, such as memory, processor, linked network and peripheral devices.

\section{Definition 2-1}

P2P network node context is a set of node context element, which is:

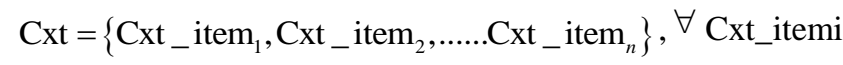

$$
\begin{aligned}
& \in \mathrm{Cxt} \text {, }
\end{aligned}
$$

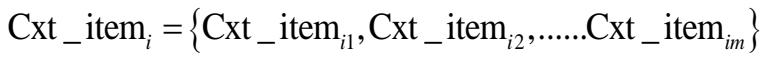

in which, Cxt_itemij is the jth history information of the $i$ th context element.

\section{Definition 2-2}

The history information of context element Cxt_item ${ }_{i j}$ is a quintuple consists of Who, Where, When, What, Why, as Cxt_item $i j=($ Who, Where, When, What, Why $)$, there into Who is the identifier of context element, where is the space information of context element, When is the time information of context element, What is the attribute information of context element, Why is the P2P workflow context semantics rules decided by context element and its history information.

For example, the speed of device context in wireless P2P network can be expressed as (the speed of device, east longitude $100^{\circ} 10^{\prime}$ northern latitude $60^{\circ} 20^{\prime}$, 2007-3-31 $10 \mathrm{~h} 15 \mathrm{~m} 30 \mathrm{~s}, 20 \mathrm{~m} / \mathrm{s}$," if the speed is decreasing, it shows that 
the device is slowing down; and accoring to the change of position we can deduct the moving direction of device").

In this paper, the metric at time $\mathrm{j}$ of the ith context element is Cxt_item ${ }_{i j}$. What.

\section{Definition 2-3}

CAN node VID (virtual Identifier) is a binary string in the path from root node to leaf node on the split tree.

VID is used in CAN struture maintenance and information routing.

\section{Definition 2-4}

CAN node coordination is the hash value coming from the key valude Key after d times of SHA-1 calulation, the $d$ is the virtual space dimensions of CAN.

For example, if CAN virtual space is d dimension, the node key value is Key, then firstly implement SHA-1 hash on Key to get the 160 digits hash value $\mathrm{H} 1$, then get the $\mathrm{H} 2$ fromH1 through SHA-1 calculation, and so on, the Hd after d times of hashing is the node coordinate value.

Node cooradinate is used in resource searching objectives in CAN.

The node context element choosen in task scheduling decision includes:

1) CAN node VID

2) CAN node coordinate

3) Transportation delay RTT (Round Trip Time): is the transportation delay from starting node to receiving node.

4) Node handling ability TPCC (Transaction Processing Performance Council): adopting the Benchmark's technical standard, performance and value measurement to test the processing unit in order to obtain the assessment parameters, usually is the TPMC Transaction per Minutes ) value.

5) Node configuration CPU: node hardware configuration especially the CPU situation.

6) Node CPU utilization ratio: the current node CPU utilization ratio is used to decide the busy extent of node.

7) Node configuration Memory: node hardware configuration Memory situation.

8) Node Memory utilizaition ratio: the current node Memory occupation situation is used to decide the remaining caculating ability of node.

9) Node assigned tasks: the assigned task quantity of the node.

10) Node OOS (Online-Offline-Status): the accessible status of the node.

\section{The Ontology Description of CAN CONTEXT Node}

Ontology is the specified description of conceptual model. Using ontology to build a model for P2P network node context can supply uniform and open sharing concept support for workflow management system.

The node context ontology built in this paper mainly includes three classes: Cxt, Vertex and Link, thereinto, Cxt is a class of universal node context ontology; Vertex is a subclass of Cxt, it presents the node context; Link is the other subclass of Cxt, it presents link context. In the execution process of workflow, there would be instances of Vertex and Link basing on real situation.

OWL based description pattern of ontology is shown in Fig. 1(without the header of OWL)

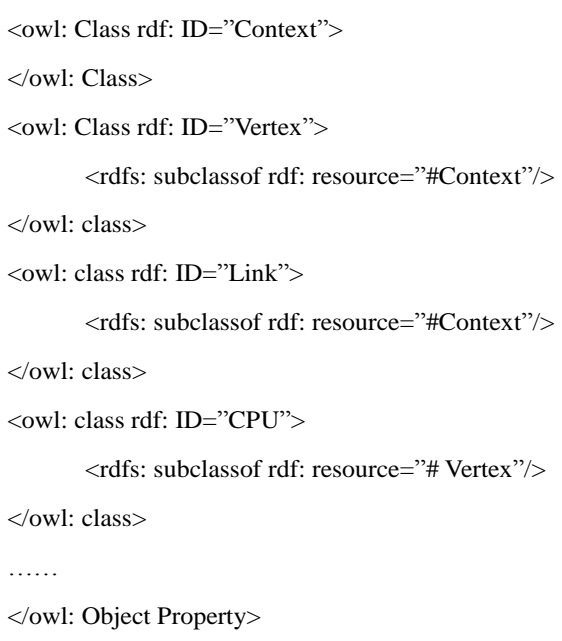

Fig. 1. OWL- based Ontology description.

To some classes, like CPU, we still need to refine the definition basing on its attributes.

For example, the OWL of CPU class, the arcs with arrow represent predicate, the end of arrow is object, the other one is subject. Subclassof is used as basic classification symbol of classes, it can interconnect a more specific class to a common class and can represent how the common class inherits from the more specific class. OnPropertyOf represents the specific attribute of class. Has Value can identify class basing on "specific" existence of attribute value, which can represent a attribute with specific value of a class instance. Xsd: string represents data type of the attribute is "string".

The depiction above illustrates that the CPU class has attribute Vendor_CPU, which has attribute value of string type; and has the attribute Model, which has attribute value of string type.

\section{The Obtaining And Management of Node Context}

There are two mechanisms to obtain P2P network node context: interval polling and instant adoption.

Interval polling is to carry out polling for specific nodes at interval of $\delta t$ by context management nodes in order to obtain context information of visited nodes.

Interval polling usually adopts centralizied management pattern, it means to choose one or several context management context, which is responsible for the global or partial centralized mangement of all nodes in the system: collecting, storing and handling the context information, and supply global or partial context information service.

Interval polling mechanism is appropriate for management nodes that need real time monitoring on specific node group, the characteristics are real time, easy to use history node information on deciding the status of specific nodes, easy to decide the semantic and the shortage is easy to cause huge network information flow, which needs to consume some handling ability of specific management nodes and inquired nodes. Fig. 2 is the algorithm of interval polling. 


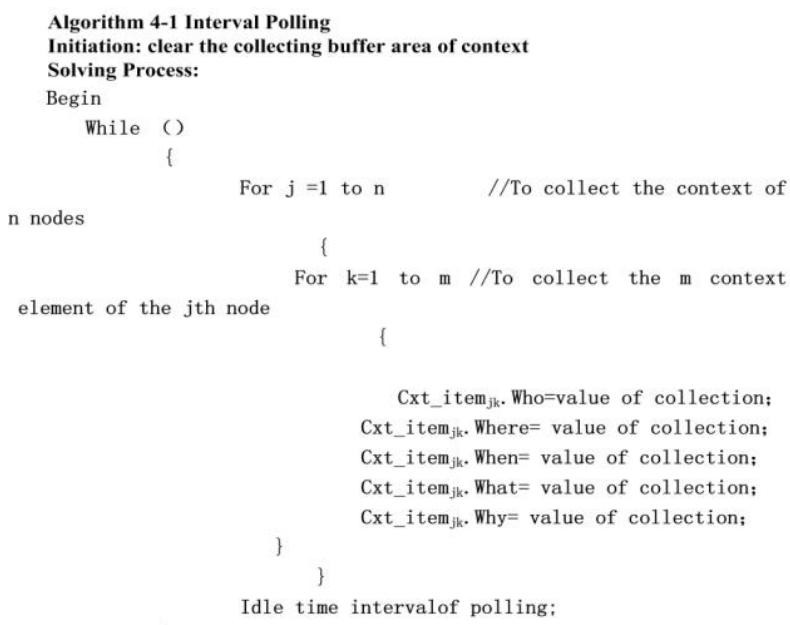

Fig. 2. Algorithm of Internal Polling.

Instant adoption is to carry out information inquiry on specific nodes by context management nodes, and the context information would be returned by inquired nodes.

The instant adoption mechanism usually is used to satisfy individual need, if the nodes need to comprehend its peripheral calculation environment, the inquired nodes would return its context information and the starting nodes would receive and use it, to the collecting results the receiving nodes decide if it would store the inquired context information based on real situation.

Instant adoption mechanism is appropriate for context management nodes that do not need real time monitoring on context situation of specific nodes, only needs to obtain instantly scenes, the characteristics is the ability of node handling and saving network bandwidth, without the need of storing space of context information, the shortage is lack of real time and context history information. Fig. 3 is the algorithm of instant adoption.

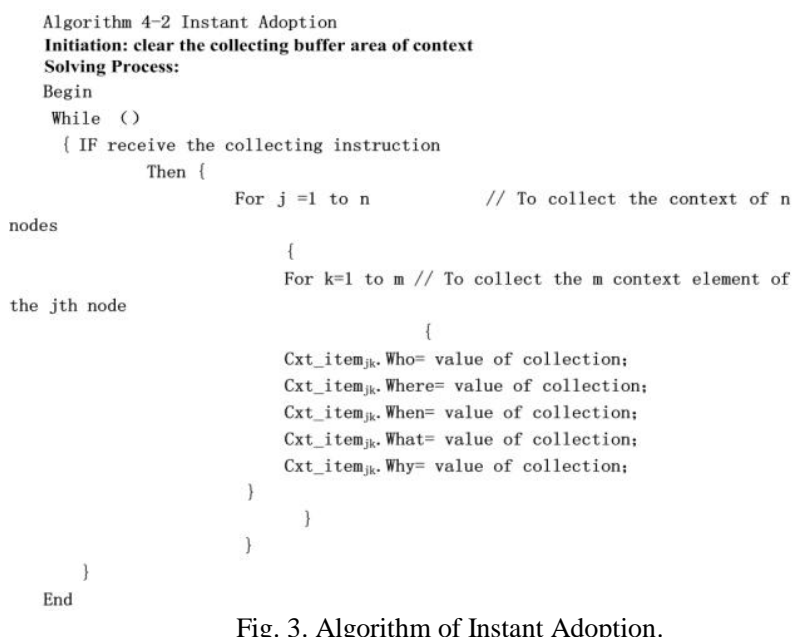

Fig. 3. Algorithm of Instant Adoption.

In the $\mathrm{P} 2 \mathrm{P}$ covering network environment, we can adopt context centralized management pattern, use the catalog management nodes to carry out centralized management of global node context information, when a node needs the context information of other nodes, we can carry out context inquiry service through centralized management nodes.

In uncentralized $\mathrm{P} 2 \mathrm{P}$ covering network environment, we can adopt uncentralized management pattern, which means to carry out inquiring request from relevant nodes through instant adoption query pattern, and the inquired nodes can supply context information.

In mixing P2P covering network environment, we can both adopt centralized management pattern and uncentralized management pattern or area centralized management pattern, it should decide based on context information need.

This paper is researching on uncentralized unstructured $\mathrm{P} 2 \mathrm{P}$ covering network, so we adopt the instant adoption query pattern.

\section{CONCLUSION}

This paper uses CAN network as the workflow environment, analyses the node context elements, gives the ontology representation of $\mathrm{P} 2 \mathrm{P}$ network node context, discusses the node context obtaining mechanism; based node context information defines the node handling ability assessment index, which is the basis of task scheduling.

\section{REFERENCES}

[1] B. W. Mitchell, "Microcomputer-based performance-monitoring and recording system for a solar heating system," Trans. of the ASAE, vol. 26, no. 5, pp. 898-901, 1983.

[2] B. W. Mitchell, "Interfacing single-board microcomputer controls to conventional controls for an environmental control system," Trans. of the ASAE, vol. 27, no. 6, pp. 1590-1594, 1984.

[3] B. W. Mitchell, "Signal conditioning for digital inputs and outputs to a microcomputer," Trans. of the ASAE, vol. 28, no. 3, pp. 949-953, 1985.

[4] X. H. Kong, "Task scheduling in network computing environments based on intelligent algorithm," Ph.D. thesis, School of Information Technology, Jiangnan University, Wuxi, China, 2007.

[5] P. Flocchini, A. Nayak, and M. Xie, "Enhancing peer-to-peer systems through redundancy," Selected Areas in Communications, IEEE Journal on Selected Areas in Communications, vol. 25, no. 1, pp. 15-24, 2007.

[6] L. Zhou, "Research on scheduling algorithm in distributed system," Ph.D. thesis, School of Computer Science and Engineering, University of Electronic Science and Technology of China, Chengdu Sichuan, China, 2008.

[7] T. Gruber, "Towards principles for the design of ontologies used for knowledge sharing," International Journal of Human-Computer Studies, vol. 43, no. 5-6, pp. 907-928, November 1995.

[8] H. T. Pu, J. J. Lin, and Y. W. Song, "Adaptive device context based mobile learning system," International Journal of Distance Education Technologies, vol. 9, no. 1, pp. 44-56, January 2011.

[9] B. Benatalhth, Q. Z. Sheng, and M. Dumas, "The Self-serv environment for web services composition," IEEE Internet Computing, vol. 7, no. 1, pp. 40-48, 2003.

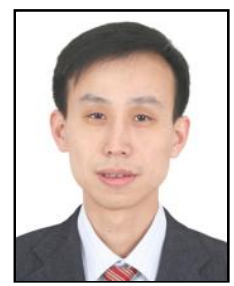

Pu Haitao was born in Taian, Shandong Province, P.R.China, on January 11,1979 . He received the Ph.D. degree in Control Theory and Control Engineering in 2011 from Shandong University of Science and Technology, Qingdao, China. His research interests include system optimization and mobile computing.

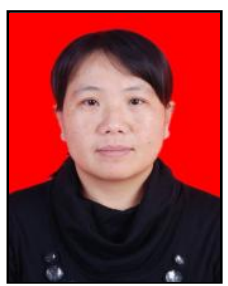

Zhang Aiying was born in Yangxin, Shandong Province, P.R.China on September, 1974. She received graduate education at Shandong University of Science and Technology from September 2003 to June 2006, and achieved master's degree in Engineering, Her major field of study is computer information processing. 


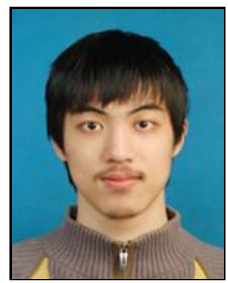

Song Enzhao was born in Shandong province, P.R.China on February 8, 1992. He is a fourth year undergraduate major in software at Beijing Jiaotong University. As a graduating student, now he practices

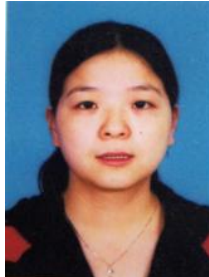

Lin Jinjiao was born in Yongkang, Zhejiang province, P.R.China on March 5, 1978. She received the Ph.D. degree in computer software and theory in Shandong University, Jinan, China, in 2008. Her research interests include database management and software theory. 